\title{
IDENTITIES FOR CONJUGATION IN THE STEENROD ALGEBRA
}

\author{
PHILIP D. STRAFFIN, JR.
}

ABSTRACT. Let $\boldsymbol{x}$ be the canonical conjugation in the Steenrod algebra $a_{2}$. I prove the identity

$$
S q^{2^{n}}+\chi\left(S q^{2^{n}}\right)=S q^{2^{n-1}} \times\left(S q^{2^{n-1}}\right)
$$

and generalizations of this identity both in $Q_{2}$ and in $Q_{p}$ where $p$ is an odd prime.

The canonical conjugation $\chi$ in the mod 2 Steenrod algebra $\mathbb{A}_{2}$ can be defined by Thom's recursion formula

$$
\sum_{i=0}^{n} S q^{i} \chi\left(S q^{n-i}\right)=0
$$

together with the stipulation that $\chi: \mathbb{Q}_{2} \rightarrow \mathbb{Q}_{2}$ be an anti-isomorphism [3]. Since the elements $S q^{2^{n}}$ multiplicatively generate $\mathbb{A}_{2}$, we can calculate $\chi$ if we can calculate $\chi\left(S q^{2}\right)$ for all $n$. The above recursion formula is unnecessarily cumbersome for this goal. In fact, the recursion can be shortened considerably by use of the following interesting

Identity. $S q^{2^{n}}+\chi\left(S q^{2^{n}}\right)=S q^{2^{n-1}} \chi\left(S q^{2^{n-1}}\right)$ for all positive integers $n$. Applying the identity recursively we obtain the

Formula.

$$
\chi\left(S q^{2^{n}}\right)=S q^{2^{n}}+\sum_{i=1}^{n-1}\left(\prod_{j=1}^{i} S q^{2^{n-j}}\right) S q^{2^{n-i}}
$$

For example,

$$
\chi\left(S q^{16}\right)=S q^{16}+S q^{8} S q^{8}+S q^{8} S q^{4} S q^{4}+S q^{8} S q^{4} S q^{2} S q^{2}
$$

In this paper, I will prove a theorem which will imply the above identity, and which also yields results about the $\bmod p$ Steenrod algebra $\mathfrak{Q}_{p}$ when $p$ is an odd prime. The technique is to use Milnor's calculation of $\chi$ in the

Received by the editors March 25, 1974.

AMS (MOS) subject classifications (1970). Primary 55G10; Secondary 05A10.

Key words and phrases. Steenrod algebra, conjugation, Milnor basis, binomial coefficients $\bmod p$. 
Milnor basis for $Q_{p}[2]$, together with properties of binomial coefficients mod $p$. This technique was noticed independently by Donald Davis, who used it to prove other identities involving $\chi$ in $\mathbb{Q}_{p}[1]$.

Let $p$ be a fixed prime. Let $R=\left(r_{1}, r_{2}, \ldots\right)$ be a sequence of nonnegative integers with only a finite number of nonzero terms. For each such $R$ there is an element $\mathcal{P} R$ in the Milnor basis for $\mathbb{Q}_{p}$, of degree $\Sigma_{i \geq 1} 2\left(p^{i}-1\right) r_{i}$ (if $p=2$, the element is written $S q^{R}$ and its degree is $\Sigma_{i \geq 1}\left(2^{i}-1\right) r_{i}$ ). We define $|R|=\Sigma_{i \geq 1} p^{i-1} r_{i}$. Then Davis' main proposition can be written.

Proposition 1. $\mathscr{P}^{m} \chi^{\left(\mathscr{P}^{n}\right)}=(-1)^{n} \Sigma_{R}\left(\begin{array}{c}|R| \\ m\end{array}\right) \mathcal{P}^{R}$ where the sum is taken over all $R$ such that $\mathcal{P}^{R}$ has the proper degree, i.e. over all $R$ such that

$$
\sum_{i \geq 1}\left(p^{i}-1\right) r_{i}=(p-1)(m+n) \text {. }
$$

If $p=2$, the only necessary modification is to write $S q$ for $\mathcal{P}$. The binomial coefficient is, of course, to be interpreted $\bmod p$.

Proof. See [1].

The one additional fact about binomial coefficients which we will need is

Proposition 2. Let $a$ and $b$ be integers. If $p^{a} \leq r \leq p^{a} b$, then

$$
\sum_{k=0}^{b}(-1)^{k}\left(\begin{array}{c}
r \\
p^{a} k
\end{array}\right) \equiv 0 \quad(\bmod p) .
$$

Proof. Write $r=p^{a} s+t$, with $1 \leq s \leq b$ and $0 \leq t<p^{\alpha}$.

Then

$$
\left(\begin{array}{c}
p^{a} s+t \\
p^{a} k
\end{array}\right) \equiv\left(\begin{array}{l}
s \\
k
\end{array}\right) \quad(\bmod p),
$$

as is easily seen by comparing the coefficients of $x^{p^{a}}$ in the congruence

$$
(1+x)^{p^{a} s+t} \equiv\left(1+x^{p^{a}}\right)^{s}(1+x)^{t} \quad(\bmod p) .
$$

Hence the proposition follows from the well-known identity $\sum_{k=0}^{b}(-1)^{k}\left(\begin{array}{l}s \\ k\end{array}\right)=0$ for $1 \leq s \leq b$.

We can now prove our main

Theorem. Let $a \geq 0$ and $b>1$ be integers. Then

$$
\sum_{k=0}^{b} \mathcal{P} p^{a}{ }^{k} \chi\left(\mathcal{P p}^{a}(b-k)\right)=0 .
$$

Examples. (1) If $a=0$, we get Thom's original recursion formula in $\mathfrak{A}_{p}$.

(2) If $p=2, a=n-1, b=2$, we get the identity at the beginning of this paper. 
(3) If $p=2, a=2, b=3$, we get

$$
\chi\left(S q^{12}\right)+S q^{4} \chi\left(S q^{8}\right)+S q^{8} \chi\left(S q^{4}\right)+S q^{12}=0 .
$$

(4) If $p=3, a=2, b=3$, we get

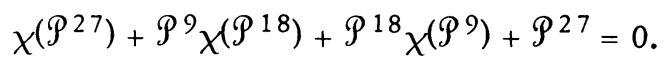

Proof of Theorem. Consider any $R=\left(r_{1}, r_{2}, \ldots\right)$ such that

$$
\sum_{i \geq 1}\left(p^{i}-1\right) r_{i}=(p-1) p^{a} b .
$$

The coefficient of $\mathcal{P} R$ in the Milnor base expansion of the sum in the Theorem is, by Proposition 1 ,

$$
\sum_{k=0}^{b}(-1)^{p^{a}(b-k)}\left(\begin{array}{c}
|R| \\
p^{a} k
\end{array}\right)=(-1)^{b} \sum_{k=0}^{b}(-1)^{k}\left(\begin{array}{c}
|R| \\
p^{a} k
\end{array}\right) .
$$

By Proposition 2, this coefficient is zero if $p^{a} \leq|R| \leq p^{a} b$. But (*) gives that

$$
|R|=\sum_{i \geq 1} p^{i-1} r_{i}=\frac{1}{p}\left[(p-1) p^{a} b+\sum_{i \geq 1} r_{i}\right]
$$

and we also have

$$
0 \leq \sum_{i \geq 1} r_{i} \leq \frac{1}{p-1} \sum_{i \geq 1}\left(p^{i}-1\right) r_{i}=p^{a} b
$$

Hence $((p-1) / p) p^{a} b \leq|R| \leq p^{a} b$; and since $b>1$, the required inequality holds.

\section{BIBLIOGRAPHY}

1. Donald Davis, The antiautomorphism of the Steenrod algebra, Proc. Amer. Math. Soc. 44 (1974), 235-236.

2. J. Milnor, The Steenrod algebra and its dual, Ann. of Math. (2) 67 (1958), 150-171. MR 20 \#6092.

3. R. Thom, Espaces fibrés en sphères et carrés de Steenrod, Ann. Sci. École Norm. Sup. (3) 69 (1952), 109-182. MR 14, 1004.

DEPARTMENT OF MATHEMATICS, BELOIT COLLEGE, BELOIT, WISCONSIN 53511 